\title{
ESTIMATIVA DA CONDUTIVIDADE ELÉTRICA DA SOLUÇÃO DO SOLO A PARTIR DO TEOR DE ÁGUA E DA CONDUTIVIDADE ELÉTRICA APARENTE DO SOLO ${ }^{1}$
}

\section{GESSIONEI DA S. SANTANA ${ }^{2}$, EUGÊNIO F. COELHO ${ }^{3}$, TIBÉRIO S. M. DA SILVA ${ }^{4}$, MÁRCIO M. RAMOS ${ }^{5}$}

\begin{abstract}
RESUMO: O crescente uso da técnica de fertirrigação não tem sido acompanhado com pesquisas para disponibilizar informações capazes de proporcionar o correto manejo dessa técnica. A reflectometria no domínio do tempo (TDR) possibilita o monitoramento simultâneo e contínuo do teor de água $(\theta)$ e da condutividade elétrica aparente do solo (CEa). A literatura apresenta diversos modelos que relacionam $\theta$ e CEa com a condutividade elétrica da solução do solo (CEw), com vistas à predição da $\mathrm{CEw}$ a partir de dados de $\theta$ e CEa obtidos por meio da técnica da TDR. Porém, muitas pesquisas demonstram a necessidade de avaliação e calibração desses modelos para solos de diferentes classes texturais. Neste trabalho, foram avaliados seis modelos com o objetivo de conhecer a capacidade dos mesmos em relacionar $\theta$, CEa e CEw. Experimentos de laboratório foram feitos, com solo de classes texturais franca e franco-arenosa, aplicando-se soluções com cinco condutividades elétricas combinadas com cinco teores de água no solo, resultando em 25 colunas de solo. Os modelos demonstraram capacidade para relacionar $\theta, \mathrm{CEa}$ e $\mathrm{CEw}$.
\end{abstract}

PALAVRAS-CHAVE: TDR, modelos matemáticos, manejo de fertirrigação.

\section{ESTIMATIVE OF SOIL SOLUTION ELECTRICAL CONDUCTIVITY FROM SOIL WATER CONTENT AND SOIL BULK ELECTRICAL CONDUCTIVITY}

\begin{abstract}
The increase of fertigation techniques has not been followed by researches that generate available information to guide a correct fertigation management. The time-domain reflectometry allows the simultaneously monitoring of soil water content $(\theta)$ and bulk electrical conductivity $(\mathrm{CEa})$. Literature presents various models which relate TDR readings of $\theta$ and CEa to soil solution electrical conductivity $(\mathrm{CEw})$. Nevertheless, many researches demonstrate the need for evaluation and calibration of these models under different soil conditions. In this work, six mathematical models were evaluated in order to establish the relationship among $\theta$, CEa e CEw. Laboratory experiments were carried out using two soil materials, by means of applying five electrical conductivities combined with five soil water contents in PVC columns. The models allowed a reasonable relationship among $\theta$, CEa e CEw with better performance of de RHOADES et al. (1976) e de VOGELER et al. (1996) models for the soils 1 and 2, respectively.
\end{abstract}

KEYWORDS: TDR, mathematical models, fertigation management.

\footnotetext{
${ }^{1}$ Extraído da tese de doutoramento do primeiro autor.

${ }^{2}$ Eng $^{-}$Agr ${ }^{\circ}$, Prof. da Escola Agrotécnica Federal de Salinas, Rodovia Salinas-Taiobeiras, km 2, Caixa Postal 71, Salinas - MG, Fone: (0XX38) 3841.1599, gessionei@gmail.com

${ }^{3}$ Eng $^{\mathrm{o}} \mathrm{Agr}^{\mathrm{o}}$, Pesquisador da Embrapa Mandioca e Fruticultura, Cruz das Almas - BA.

${ }^{4} \mathrm{Eng}^{\mathrm{O}} \mathrm{Agr}^{\mathrm{o}}$, M.Sc., Autônomo, Cruz das Almas - BA.

${ }^{5}$ Eng $^{\mathrm{o}}$ Agr $^{\mathrm{o}}$, Prof. da Universidade Federal de Viçosa - DEA, Viçosa - MG.

Recebido pelo Conselho Editorial em: 4-1-2005

Aprovado pelo Conselho Editorial em: 30-8-2006
} 


\section{INTRODUÇÃO}

A aplicação de fertilizantes via água de irrigação (fertirrigação) tem crescido substancialmente em pólos agrícolas irrigados, devido, principalmente, às vantagens conferidas por essa técnica e ao incremento no uso de equipamentos de irrigação localizada. Dentre as vantagens da fertirrigação, destacam-se a possibilidade de parcelamento mais adequado das doses de nutrientes, que resulta em maior sintonia da aplicação de fertilizantes com a marcha de absorção de nutrientes das culturas, em maior aproveitamento dos nutrientes pelas plantas e na obtenção de plantas nutridas de maneira mais adequada; economia de mão-de-obra e redução do tráfego de pessoas e máquinas na lavoura (PINTO, 2001). Por sua vez, a irrigação localizada possibilita, de modo geral, a aplicação de água e fertilizantes com maior eficiência.

Apesar de a fertirrigação se mostrar promissora, seu êxito está condicionado à geração de informações capazes de proporcionar o seu manejo de forma mais adequada. O monitoramento de íons no solo constitui-se em uma das principais ferramentas no manejo de fertirrigação. Esse tem sido realizado com base em amostragens de solo ou de solução do solo, por meio de extratores (RHOADES \& OSTER, 1986).

A amostragem de solo, além de ter o inconveniente de destruir a estrutura do solo, é limitada no tempo e no espaço, uma vez que seus resultados representam uma condição iônica referente apenas ao momento e ao volume de solo em que foi realizada. De forma análoga, a extração de solução do solo também não permite o conhecimento do estado iônico do solo em tempo real, além de só ser possível em regiões do solo com teores de água associados a potenciais superiores àqueles aplicados nos extratores. Portanto, a implementação de método capaz de monitorar de forma contínua a distribuição de água e íons em campos agrícolas faz-se necessária para facilitar o manejo da fertirrigação nos solos cultivados.

A técnica da Reflectometria no Domínio do Tempo (TDR) foi inicialmente aplicada ao solo por TOPP \& DAVIS (1980) com o objetivo de medir o teor de água no solo, com base no tempo de deslocamento de pulsos eletromagnéticos por uma linha de transmissão (sonda) introduzida no solo, composta de hastes de aço de comprimento conhecido (TOMMASELLI \& BACCHI, 2001).

Uma alternativa para o monitoramento do estado iônico do solo, visando ao correto manejo da fertirrigação, é a técnica da TDR que, além de possibilitar, com o uso de uma única sonda, a determinação simultânea do teor de água $(\theta)$ e da condutividade elétrica aparente do solo (CEa), em tempo real, de formas contínua, automática e rápida, e com o mínimo distúrbio da estrutura do solo, viabiliza, de forma indireta, o conhecimento da condutividade elétrica da solução do solo (CEw) (DALTON et al., 1984; WRAITH \& DAS, 1998; NOBORIO, 2001; MUÑOZ-CARPENA et al., 2001).

A literatura tem apresentado diversos modelos que relacionam $\theta$, CEa e CEw (RHOADES et al., 1976; RHOADES et al., 1989; NADLER et al., 1984, modificado por RHOADES et al., 1989; MUALEN \& FRIEDMAN, 1991; HEIMOVAARA et al., 1995; VOGELER et al., 1996).

A técnica da TDR tem sido utilizada com sucesso em várias pesquisas com o objetivo de determinar $\theta$ e a CEa (RHOADES et al., 1976; RHOADES et al., 1989; HEIMOVAARA et al., 1995; VOGELER et al., 1996; WRAITH \& DAS, 1998; MUÑOZ-CARPENA et al. 2001; PERSSON \& UVO, 2003), a modelagem do bulbo molhado (SOUZA, 2002), a modelagem da dinâmica de água e solutos na zona radicular (MMOLAWA \& OR, 2000), a identificação do perfil de extração de água pelo sistema radicular das culturas (COELHO \& OR, 1996) e a estimativa da evapotranspiração das culturas (WARD \& DUNIN, 2001).

Neste trabalho, teve-se o objetivo de avaliar alguns modelos disponíveis na literatura quanto à capacidade de relacionar $\theta, \mathrm{CEa}$ e $\mathrm{CEw}$, a fim de possibilitar a estimativa de $\mathrm{CEw}$ a partir de dados de $\theta$ e CEa, obtidos por meio da técnica da TDR. 


\section{MATERIAL E MÉTODOS}

O solo utilizado no experimento foi coletado no município de Nova Porteirinha, que se localiza na região norte de Minas Gerais. A coleta do solo foi feita em camadas de 0,20 m de espessura, a partir da superfície do solo até $0,80 \mathrm{~m}$ de profundidade, o que possibilitou definir duas classes texturais para serem estudadas (Tabela 1). O solo coletado na camada superficial $(0,0$ $0,20 \mathrm{~m})$ resultou na classe textural franco-arenosa $\left(\mathrm{CT}_{\mathrm{fa}}\right)$, ao passo que o solo coletado na profundidade de 0,20-0,80 m resultou na classe textural franca $\left(\mathrm{CT}_{\mathrm{f}}\right)$. As características químicas do solo das duas classes texturais estudadas são apresentadas na Tabela 2. O solo foi seco ao ar, destorroado e passado em peneira com malha de $2 \mathrm{~mm}$. Segmentos de tubo de PVC com 0,19 m de altura e $0,098 \mathrm{~m}$ de diâmetro interno foram preenchidos com 1,9 kg de terra fina seca ao ar (TFSA), até a altura de $0,17 \mathrm{~m}$, perfazendo volume de solo no vaso de $0,00128231 \mathrm{~m}^{3}$.

TABELA 1. Características físico-hídricas das classes texturais do solo estudado.

\begin{tabular}{|c|c|c|c|c|c|c|c|}
\hline Classe Textural & Areia & Silte & Argila & $\begin{array}{c}\text { Densidade do } \\
\text { Solo }\end{array}$ & $\begin{array}{c}\text { Densidade de } \\
\text { Partículas }\end{array}$ & \multicolumn{2}{|c|}{ Teor de Água no Solo } \\
\hline & & & & & & $-10 \mathrm{kPa}$ & $-1.500 \mathrm{kPa}$ \\
\hline & \multicolumn{3}{|c|}{$\left(\mathrm{g} \mathrm{kg}^{-1}\right)$} & \multicolumn{2}{|c|}{$\mathrm{kg} \mathrm{dm}^{-3}$} & \multicolumn{2}{|c|}{$\left(\mathrm{kg} \mathrm{kg}^{-1}\right)$} \\
\hline Franca & 427,3 & 417,3 & 155,3 & 1,47 & 2,39 & 0,2226 & 0,1608 \\
\hline Franco-arenosa & 544,0 & 355,0 & 101,0 & 1,45 & 2,43 & 0,2013 & 0,1503 \\
\hline
\end{tabular}

TABELA 2. Características químicas das classes texturais do solo estudado.

\begin{tabular}{|c|c|c|c|c|c|c|c|c|c|c|c|c|c|}
\hline & $\mathrm{pH}$ & $\mathrm{P}$ & K & $\mathrm{Ca}$ & $\mathrm{Mg}$ & $\mathrm{Ca}+\mathrm{Mg}$ & $\mathrm{Al}$ & $\mathrm{Na}$ & $\mathrm{H}+\mathrm{Al}$ & $\mathrm{S}$ & CTC & $\mathrm{V}$ & M.O. \\
\hline Cla & $\begin{array}{l}\text { em } \\
\text { água }\end{array}$ & $\begin{array}{c}\mathrm{mg}^{-3} \\
\mathrm{dm}^{-3}\end{array}$ & \multicolumn{9}{|c|}{$\mathrm{cmol}_{\mathrm{c}} \mathrm{dm}$} & $\%$ & \\
\hline & 6,6 & 24,0 & 0,59 & $\overline{5,6}$ & 0,1 & 5,7 & 0 , & 9 & 0,88 & 6,43 & 7,31 & 88,0 & 3,47 \\
\hline Franco-arenosa & 6,5 & 23,0 & 0,20 & 6,1 & 0,3 & 6,4 & 0,1 & 0,08 & 1,54 & 6,68 & 8,22 & 81,3 & 5,68 \\
\hline
\end{tabular}

O experimento consistiu de 25 tratamentos (colunas de solo) resultantes da combinação de cinco teores de água no solo $(\theta)$, com cinco condutividades elétricas $(\mathrm{CE})$, obtidas a partir da aplicação de soluções com cinco concentrações de cloreto de potássio $(\mathrm{KCl})$ e de cinco volumes dessas soluções para obter cinco $\theta$. Esses tratamentos foram repetidos duas vezes, para a $\mathrm{CT}_{\mathrm{f}}$, resultando em 50 observações, enquanto para a $\mathrm{CT}_{\mathrm{fa}}$ não houve repetição; portanto, obtiveram-se apenas 25 observações. Os $\theta$ foram correspondentes a 20;40;60; 80 e 100\% da água disponível e as CE das soluções correspondentes a 1,0; 2,5; 4,0; 5,5 e 7,0 dS m${ }^{-1}$.

O teor de água residual no solo $\left(\theta_{\mathrm{r}}\right)$ foi obtido pelo método gravimétrico-padrão de estufa, possibilitando a correta complementação de $\theta$ para os níveis desejados.

Preliminarmente, um ensaio foi realizado para determinar a relação entre concentrações de cloreto de potássio $(\mathrm{KCl})$ e condutividades elétricas $(\mathrm{CE})$ de soluções de $\mathrm{KCl}$. Submetendo-se os valores de concentrações de $\mathrm{KCl}$ e $\mathrm{CE}$ das soluções de $\mathrm{KCl}$ à análise de regressão, obteve-se a seguinte expressão:

$$
(\mathrm{KCl})=\frac{\mathrm{CE}-0,0900}{1,6257} \quad \mathrm{R}^{2}=1,00
$$

em que,

$\mathrm{KCl}$ e $\mathrm{CE}$ - correspondem, respectivamente, às concentrações de cloreto de potássio nas soluções, em $\mathrm{g} \mathrm{L}^{-1}$, e à condutividade elétrica das soluções de cloreto de potássio, em dS m$~^{-1}$. 
Com base na eq.(1), pesaram-se quantidades de $\mathrm{KCl}$ que foram diluídas em água destilada, obtendo-se as soluções que foram aplicadas sobre a superfície do solo nas colunas de PVC. Em seguida, o solo foi coberto e mantido em repouso por 24 horas, a fim de evitar perda de água por evaporação e de propiciar distribuição mais uniforme da solução em toda a coluna de solo. Em seguida, efetuaram-se as leituras de $\theta$ e CEa por meio da TDR, utilizando uma única sonda, com três hastes de $0,10 \mathrm{~m}$ de comprimento. Simultaneamente, leituras de temperatura foram feitas com termômetro instalado em uma coluna de solo.

O teor de água no solo $(\theta)$ foi estimado por meio da equação de LEDIEU et al. (1986) [eq.(2)] e, posteriormente, corrigido de acordo com as equações de calibração da TDR para leitura de $\theta$, para o solo da área experimental [eqs.(3a) e (3b)].

$$
\begin{array}{ll}
\theta=0,1138 \sqrt{\varepsilon_{\mathrm{b}}}-0,1758 & \\
\theta=-0,1722+0,0350 \varepsilon_{\mathrm{b}}-0,0013 \varepsilon_{\mathrm{b}}{ }^{2}+2,010^{-5} \varepsilon_{\mathrm{b}}{ }^{3} & \text { para a } \mathrm{CT}_{\mathrm{fa}} \\
\theta=-0,1309+0,0250 \varepsilon_{\mathrm{b}}-0,0006 \varepsilon_{\mathrm{b}}{ }^{2}+9,010^{-6} \varepsilon_{\mathrm{b}}{ }^{3} & \text { para a } \mathrm{CT}_{\mathrm{f}}
\end{array}
$$

em que,

$\theta$ - teor de água no solo, $\mathrm{cm}^{3} \mathrm{~cm}^{-3}, \mathrm{e}$
$\varepsilon_{\mathrm{b}}$ - constante dielétrica do solo.

A CEa foi estimada por meio da equação de GIESE \& TIEMANN (1975) [eq.(4)] e, posteriormente, teve seus valores corrigidos para a temperatura de $25^{\circ} \mathrm{C}$, conforme eq.(5) (RICHARDS, 1954):

$$
\mathrm{CEa}=\frac{\mathrm{Z}_{0}}{120 \pi \mathrm{L} \mathrm{Z}}\left(\frac{2 \mathrm{~V}_{0}}{\mathrm{~V}_{\mathrm{inf}}}-1\right)
$$

em que,

CEa - condutividade elétrica aparente do solo, $\mathrm{dS} \mathrm{m}^{-1}$;

L - comprimento da sonda, m;

$\mathrm{Z}_{0}$ - impedância característica da sonda, obtida em água destilada, conforme SOUZA et. al. (2004);

$\mathrm{Z}_{\mathrm{u}}$ - impedância no cabo, $\sim 50 \Omega$;

$\mathrm{V}_{0}$ - amplitude do sinal eletromagnético gerado pelo TDR, e

$\mathrm{V}_{\text {inf }}$ - amplitude do sinal eletromagnético após a reflexão no início da sonda.

$$
\mathrm{f}_{\mathrm{T}}=1+\frac{(25-\mathrm{T})}{49,7}+\frac{(25-\mathrm{T})^{2}}{3728} \quad \text { para } 20 \leq \mathrm{T} \leq 47^{\circ} \mathrm{C}
$$

em que,

$\mathrm{f}_{\mathrm{T}}$ - fator de correção da CE quanto aos efeitos da temperatura, adimensional, e

$\mathrm{T}$ - temperatura do meio no qual a sonda de TDR se encontra inserida, ${ }^{\circ} \mathrm{C}$.

Logo após as leituras de $\theta$ e CEa com a TDR, a sonda foi retirada e substituída por um segmento de tubo PVC de $12 \mathrm{~mm}$ de diâmetro até a profundidade correspondente ao comprimento das hastes da sonda. No orifício feito pelo tubo de PVC, introduziu-se um extrator de solução do solo, devidamente limpo, e aplicou-se uma sucção de $70 \mathrm{kPa}$ (RHOADES et al., 1976; HEIMOVAARA et al., 1995; WRAITH \& DAS, 1998; VOGELER et al., 1996; MUÑOZCARPENA et al., 2001). Após a extração de aproximadamente $15 \mathrm{~mL}$ de solução do solo, retirouse o extrator, homogeneizou-se a solução, armazenando-a em frascos de vidro previamente limpos para evitar contaminação por outros íons, determinando-se, posteriormente, a CEw por meio de condutivímetro de mesa. 
Os dados de $\theta$, CEa e CEw foram processados, visando à obtenção da relação existente entre essas variáveis e dos parâmetros dos seis modelos avaliados neste estudo (Tabela 3).

TABELA 3. Modelos avaliados no estudo ${ }^{1}$.

\begin{tabular}{|c|c|c|}
\hline Autores & Modelo & Parâmetros $^{2}$ Equação $\mathrm{n}^{\mathrm{o}}$ \\
\hline $\begin{array}{l}\text { RHOADES et al. (1976) } \\
\text { (19) }\end{array}$ & $\mathrm{CEa}=\left(\mathrm{a} \theta^{2}+\mathrm{b} \theta\right) \mathrm{CEw}+\mathrm{CEs}$ & $\mathrm{a}, \mathrm{b}, \mathrm{CEs}^{3}$ \\
\hline $\begin{array}{l}\text { NADLER et al. (1984), } \\
\text { modificado por RHOADES }\end{array}$ & $\mathrm{CEW}=\mathrm{f}_{\mathrm{T}}\left(\mathrm{CE}_{\mathrm{a}}-\delta \mathrm{CEs}\right) \mathrm{F}(\theta)$ & $\mathrm{CEs}^{3}, \phi_{\mathrm{s}}, \theta_{\mathrm{ws}}$ \\
\hline
\end{tabular}

et al. $(1989)^{4}$

RHOADES et al. (1989)

$$
\mathrm{CEa}=[\theta-(\mathrm{c} \theta+\mathrm{d})] \mathrm{CEw}+\frac{\left(\theta_{\text {sol }}+\mathrm{c} \theta+\mathrm{d}\right)^{2}}{\theta_{\text {sol }}} \quad \mathrm{c}, \mathrm{d}
$$

MUALEN \& FRIEDMAN (1991)

$$
\mathrm{CEa}=\mathrm{CEw}\left(\frac{\theta^{\beta}}{\theta \mathrm{s}}\right)+\mathrm{CEs} \quad \beta, \mathrm{CEs}^{3}
$$

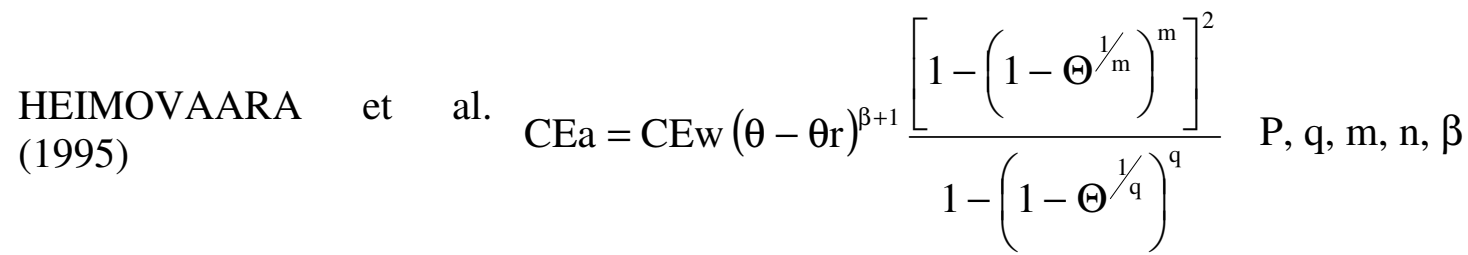

VOGELER et al. (1996) $\quad \mathrm{CEa}=(\mathrm{c} \theta-\mathrm{d}) \mathrm{CEw}+(\mathrm{a} \theta-\mathrm{b})$

a, b, c, d

${ }^{1}$ sendo CEa, CEw e CEs em dS m${ }^{-1}$ e $\theta, \theta$ s e $\theta \mathrm{r} \mathrm{em} \mathrm{cm}^{3} \mathrm{~cm}^{-3}$. No modelo de NADLER et al. (1984), modificado por RHOADES et al. (1989), CEa, CEw e CEs devem estar em S m ${ }^{-1} ;{ }^{2}$ Obtidos por otimização; ${ }^{3}$ Corresponde à condutividade elétrica da fração sólida do solo. Está associada com as trocas iônicas na interface sólido-líquido do solo e é normalmente considerada como uma constante para cada solo; ${ }^{4}$ Esse modelo já apresenta na sua estrutura o fator de correção da CE quanto ao efeito da temperatura $\left(\mathrm{f}_{\mathrm{T}}\right)$, conforme metodologia do U. S. SALINITY LABORATORY STAFF (1954).

A fração volumétrica do solo ocupada por solutos $\left(\theta_{\text {sol }}\right)$ foi obtida por meio da eq.(12).

$$
\theta_{\text {sol }}=\frac{\mathrm{Ds}}{\mathrm{Dp}}
$$

em que, Ds e Dp correspondem, respectivamente, à massa específica do solo e das partículas, $\mathrm{kg} \mathrm{dm}^{-3}$.

O índice de saturação $(\Theta)$ foi calculado conforme eq.(13).

$$
\Theta=\frac{\left(\theta-\theta_{\mathrm{r}}\right)}{\left(\theta_{\mathrm{s}}-\theta_{\mathrm{r}}\right)}
$$

em que, $\theta_{\mathrm{r}}$ e $\theta_{\mathrm{s}}$ correspondem, respectivamente, aos teores de água no solo residual e de saturação, $\mathrm{cm}^{3} \mathrm{~cm}^{-3}$.

O $\theta_{\mathrm{s}}$ foi obtido por meio da equação de cálculo da porosidade total do solo [eq.(14)].

$$
\theta_{\mathrm{s}}=1-\left(\frac{\mathrm{Ds}}{\mathrm{Dp}}\right)
$$

O parâmetro empírico $(\delta)$ e o fator $\mathrm{F}(\theta)$, que prevêem o efeito da tortuosidade dos poros do solo sobre o fluxo de corrente elétrica no meio poroso, foram obtidos por meio das eqs.(15) e (16), respectivamente, válidas para 0,2 $<\mathrm{CEws}<0,4 \mathrm{~S} \mathrm{~m}^{-1}$ e CEs $<0,15 \mathrm{~S} \mathrm{~m}^{-1}$ (RHOADES et al., 1989). As CEws correspondem à condutividade elétrica da água higroscópica do solo. 


$$
\begin{aligned}
& \delta=\frac{\left(\varphi_{\mathrm{s}}+\theta_{\mathrm{ws}}\right)^{2}}{\varphi_{\mathrm{s}}} \\
& \mathrm{F}(\theta)=\frac{1}{\left(\theta-\theta_{\mathrm{ws}}\right)}
\end{aligned}
$$

em que,

$\phi_{\mathrm{s}}$ - conteúdo volumétrico da fração sólida do solo, $\mathrm{m}^{3} \mathrm{~m}^{-3}$, e

$\theta_{\mathrm{ws}}$ - água higroscópica do solo, $\mathrm{m}^{3} \mathrm{~m}^{-3}$.

Os parâmetros empíricos $m$ e $q$ foram calculados por meio das eqs.(17) e (18), respectivamente (HEIMOVAARA et al., 1995).

$$
\begin{aligned}
& \mathrm{m}=1-\left(\frac{1}{\mathrm{n}}\right) \\
& \mathrm{q}=1-\left(\frac{2}{\mathrm{p}}\right)
\end{aligned}
$$

A avaliação dos modelos, quanto à sua capacidade de estimar a $\mathrm{CEw}$, a partir de dados de $\theta \mathrm{e}$ $\mathrm{CEa}$, obtidos com a técnica da TDR, foi feita com base no coeficiente de determinação $\left(\mathrm{R}^{2}\right)$ e no grau de ajuste da variável dependente $(\mathrm{Y})$ com a variável independente $(\mathrm{X})$, mediante ajuste linear $\mathrm{Y}=\mathrm{aX}$, após otimização de seus ajustes aos dados medidos, por meio de planilha eletrônica.

\section{RESULTADOS E DISCUSSÃO}

Os parâmetros dos modelos avaliados e os respectivos coeficientes de determinação $\left(\mathrm{R}^{2}\right)$ são apresentados nas Tabelas 4 e 5 , referentes às classes texturais franca $\left(\mathrm{CT}_{\mathrm{f}}\right)$ e franco-arenosa $\left(\mathrm{CT}_{\mathrm{fa}}\right)$, respectivamente. Verifica-se que, para ambas as classes texturais, os modelos de RHOADES et al. (1976), VOGELER et al., (1996) e MUALEN \& FRIEDMAN (1991) foram os que melhor se ajustaram aos dados obtidos, com $\mathrm{R}^{2}$ superiores a 0,80. O modelo de HEIMOVAARA et al. (1995) não se ajustou bem aos dados observados $\left(\mathrm{R}^{2}\right.$ iguais a 0,6209 e 0,4026, para as $\mathrm{CT}_{\mathrm{f}}$ e $\mathrm{CT}_{\mathrm{fa}}$, respectivamente). No trabalho de MUÑOZ-CARPENA et al. (2001), em que a relação entre CEa, CEw e $\theta$ foi avaliada, mediante a aplicação de soluções de BrK no solo, o modelo linear de RHOADES et al. (1976) foi o que melhor explicou essa relação, comparativamente aos modelos de VOGELER et al., (1996) e RHOADES et al. (1989). MUÑOZ-CARPENA et al. (2001) relatam que o modelo de RHOADES et al. (1976) também tem se destacado em outros trabalhos, tal como no de DE NEVE et al. (2000), e mencionam, ainda, que o menor número de parâmetros desse modelo faz com que não haja justificativa para o uso de outros modelos com maior número de parâmetros.

WRAITH \& DAS (1998), aplicando soluções de $\mathrm{KNO}_{3}$ no solo, avaliaram a relação entre CEa, CEw e $\theta$ por meio do modelo de RHOADES et al. (1976) e obtiveram bom ajuste desse modelo aos dados experimentais. É importante frisar que, apesar de o modelo empírico de VOGELER et al. (1996) ter sido concebido para solos com elevada agregação, esse se ajustou bem aos dados, com $\mathrm{R}^{2}$ próximo daquele obtido pelo modelo de RHOADES et al. (1976). O modelo de MUALEN \& FRIEDMAN (1991) também dispõe de apenas dois parâmetros e, no entanto, ajustouse bem aos dados ( $\mathrm{R}^{2}$ igual a 0,8077$)$.

Quanto ao parâmetro CEs, presente nos modelos de RHOADES et al. (1976), NADLER et al. (1984), modificado por RHOADES et al. (1989) e MUALEN \& FRIEDMAN (1991), percebe-se que seus valores foram relativamente concordantes para os modelos de RHOADES et al. (1976) e MUALEN \& FRIEDMAN (1991) $\left(0,1534\right.$ e $0,1456 \mathrm{dS} \mathrm{m}^{-1}$ para a $\mathrm{CT}_{\mathrm{f}}$, e 0,1643 e $0,1709 \mathrm{dS} \mathrm{m}^{-1}$ para a $\mathrm{CT}_{\text {fa }}$, respectivamente). Resultados similares a esses foram obtidos por PERSSON \& UVO (2003), ajustando os modelos de RHOADES et al. (1976) e MUALEN \& FRIEDMAN (1991) aos 
dados de $\theta$, CEa e CEw, em solo com características texturais semelhantes às do solo estudado neste trabalho. Já o modelo de NADLER et al. (1984), modificado por RHOADES et al. (1989),

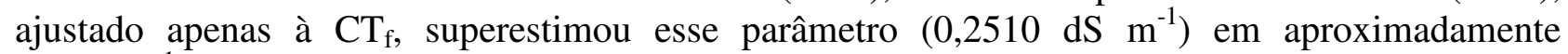
$0,1 \mathrm{dS} \mathrm{m}^{-1}$, comparativamente aos outros dois modelos.

TABELA 4. Parâmetros e coeficientes de determinação dos modelos ajustados aos dados de $\theta$, CEa e CEw, para a $\mathrm{CT}_{\mathrm{f}}$

\begin{tabular}{|c|c|c|c|c|c|c|c|c|c|c|c|}
\hline \multirow{2}{*}{ Modelo/Autor } & \multicolumn{10}{|c|}{ Parâmetros } & \multirow{2}{*}{$\mathrm{R}^{2}$} \\
\hline & $\mathrm{a}$ & $\mathrm{b}$ & $\mathrm{c}$ & $\mathrm{d}$ & $\mathrm{CEs}^{1}$ & $\phi_{\mathrm{s}}$ & $\theta_{\mathrm{ws}}$ & $\mathrm{p}$ & $\mathrm{n}$ & $\beta$ & \\
\hline RHOADES et al. (1976) & 6,0261 & $-0,3403$ & - & - & 0,1534 & - & - & - & - & - & 0,8311 \\
\hline VOGELER et al. (1996) & 1,0135 & 0,0675 & 1,4080 & 0,0891 & - & - & - & - & - & - & 0,8277 \\
\hline MUALEN \& FRIEDMAN (1991) & - & - & - & - & 0,1456 & - & - & - & - & 1,6378 & 0,8077 \\
\hline RHOADES et al. (1989) & - & - & $-0,7965$ & $-0,1626$ & - & - & - & - & - & - & 0,7437 \\
\hline HEIMOVAARA et al. (1995) & - & - & - & - & - & - & - & $-5,6518$ & 10.000 & $-0,8268$ & 0,6209 \\
\hline NADLER et al. (1984), mod. por & - & - & - & - & 0,0251 & 0,6000 & 0,0010 & - & - & - & 0,6054 \\
\hline
\end{tabular}
RHOADES et al. (1989)

${ }^{1}$ sendo, CEs, em dS m${ }^{-1}$, exceto para o modelo de NADLER et al. (1984), modificado por RHOADES et al. (1989), em que, CEs é expressa em $\mathrm{S} \mathrm{m}^{-1}$.

TABELA 5. Parâmetros e coeficientes de determinação dos modelos ajustados aos dados de $\theta$, CEa e $\mathrm{CEw}$, para a $\mathrm{CT}_{\mathrm{fa}}$

\begin{tabular}{|c|c|c|c|c|c|c|c|c|c|c|c|}
\hline \multirow{2}{*}{ Modelo/Autor } & \multicolumn{10}{|c|}{ Parâmetros } & \multirow{2}{*}{$\mathrm{R}^{2}$} \\
\hline & $\mathrm{a}$ & $\mathrm{b}$ & $\mathrm{c}$ & $\mathrm{d}$ & $\mathrm{CEs}^{1}$ & $\phi_{\mathrm{s}}$ & $\theta_{\mathrm{ws}}$ & $\mathrm{p}$ & $\mathrm{n}$ & $\beta$ & \\
\hline VOGELER et al. (1996) & 2,1558 & 0,2821 & $-0,6625$ & $-0,4227$ & - & - & - & - & - & - & 0,8504 \\
\hline RHOADES et al. (1976) & 1,5911 & 0,9835 & - & - & 0,1643 & - & - & - & - & - & 0,8123 \\
\hline MUALEN \& FRIEDMAN (1991) & - & - & - & - & 0,1709 & - & - & - & - & 1,4839 & 0,8109 \\
\hline RHOADES et al. (1989) & - & - & $-0,9629$ & $-0,1235$ & - & - & - & - & - & - & 0,7537 \\
\hline HEIMOVAARA et al. (1995) & - & - & - & - & - & - & - & -169.493 & 10.000 & $-0,7593$ & 0,4026 \\
\hline
\end{tabular}

${ }^{1}$ sendo, CEs, em dS m${ }^{-1}$, exceto para o modelo de NADLER et al. (1984), mod. por RHOADES et al. (1989), em que, CEs é expressa em $\mathrm{S} \mathrm{m}^{-1}$.

A CEs que corresponde à condutividade elétrica da fração sólida do solo é um parâmetro que está associado com as trocas iônicas na interface sólido-líquido do solo (MUÑOZ-CARPENA et al., 2001). Portanto, a estimativa de maior CEs sugere maior potencial de passagem de íons salinos da fração sólida para a fração líquida do solo, resultando, assim, em maior CEw. Os valores de CEs obtidos pelos modelos de RHOADES et al. (1976) e MUALEN \& FRIEDMAN (1991) para a CT $_{\text {fa }}$ foram ligeiramente maiores que aqueles obtidos pelos mesmos modelos, para a $\mathrm{CT}_{\mathrm{f}}$. Essa constatação reforça os relatos de PONIZOVSKY et al. (1999) e JACOBSEN \& SCHJONNING (1993) de que a textura do solo tem influência nos parâmetros de calibração da TDR.

O baixo desempenho do modelo de HEIMOVAARA et al. (1995) aos dados observados pode decorrer do fato de esse modelo não conter na sua estrutura o parâmetro CEs. Ademais, esse modelo fundamenta-se na curva de retenção de água no solo, que pode variar com o solo, resultando, por conseguinte, em limitação para esse modelo.

Nas Figuras 1 e 2, referentes às $\mathrm{CT}_{\mathrm{f}}$ e $\mathrm{CT}_{\mathrm{fa}}$, respectivamente, traçaram-se, para cada $\theta$, os valores dos dados medidos e estimados pelos modelos, utilizando os parâmetros contidos nas Tabelas 4 e 5. Observa-se que a CEw varia linearmente com a CEa. Essa linearidade também foi observada em outras pesquisas (RHOADES et al., 1976; DALTON et al., 1984; VOGELER et al., 1997; NADLER, 1997; MMOLAWA \& OR, 2000; MUÑOZ-CARPENA et al., 2001). Verifica-se que, para um mesmo valor de $\mathrm{CEa}$, a $\mathrm{CEw}$ é tanto menor quanto maior for o teor de água no solo, o que se deve à maior diluição dos sais presentes na solução do solo. Por outro lado, percebe-se que, para um mesmo valor de $\mathrm{CEw}$, quanto maior o teor de água no solo, maior a $\mathrm{CEa}$, o que se justifica pelo fato de a CEa se referir à condutividade elétrica do meio como um todo, computando, também, a condutividade elétrica proveniente das trocas iônicas na interface sólido-líquido (CEs). 

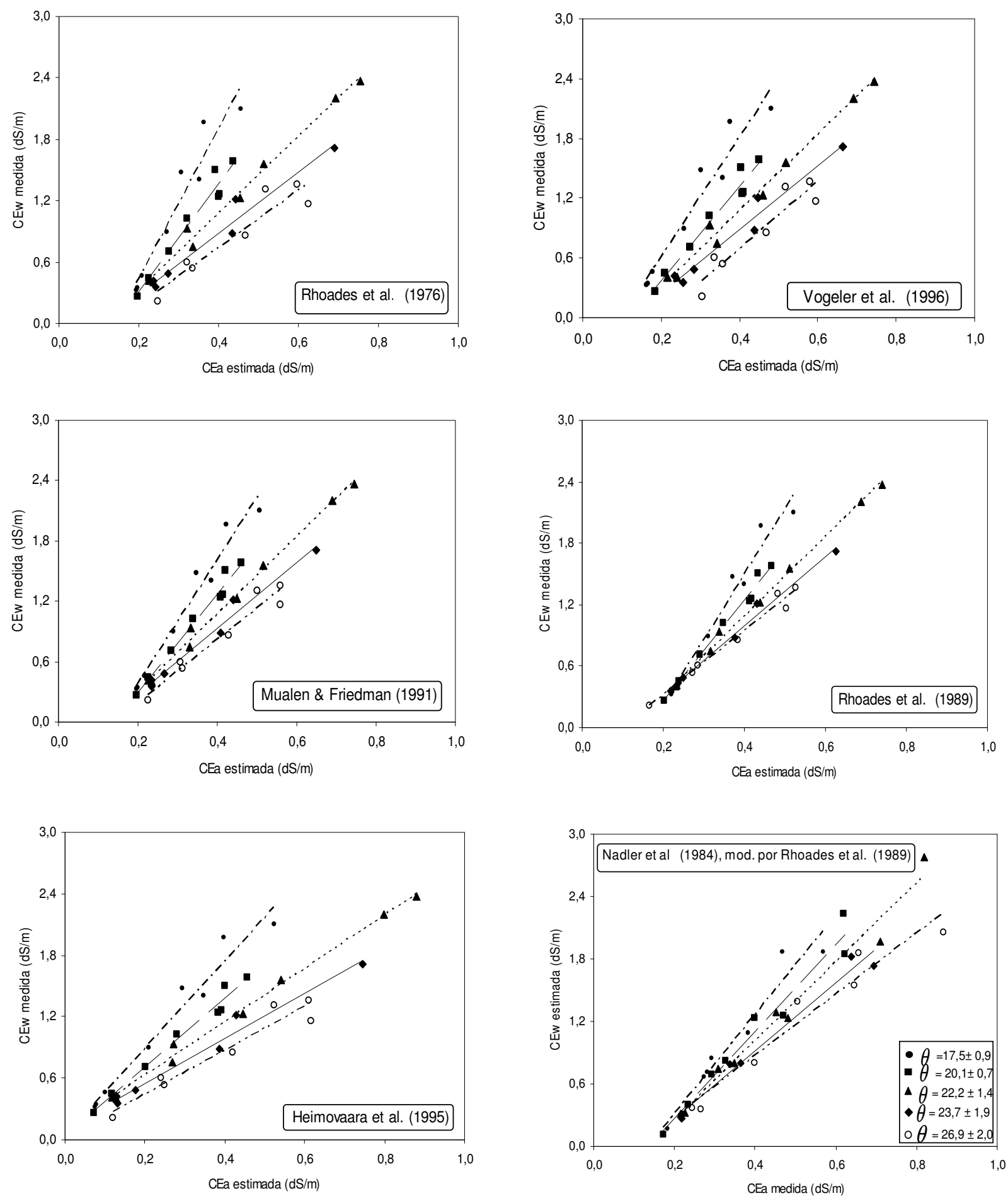

FIGURA 1. Relação entre CEa e CEw obtida a partir dos ajustes dos modelos que relacionam CEa, $\mathrm{CEw}$ e $\theta$, para a $\mathrm{CT}_{\mathrm{f}}$. 

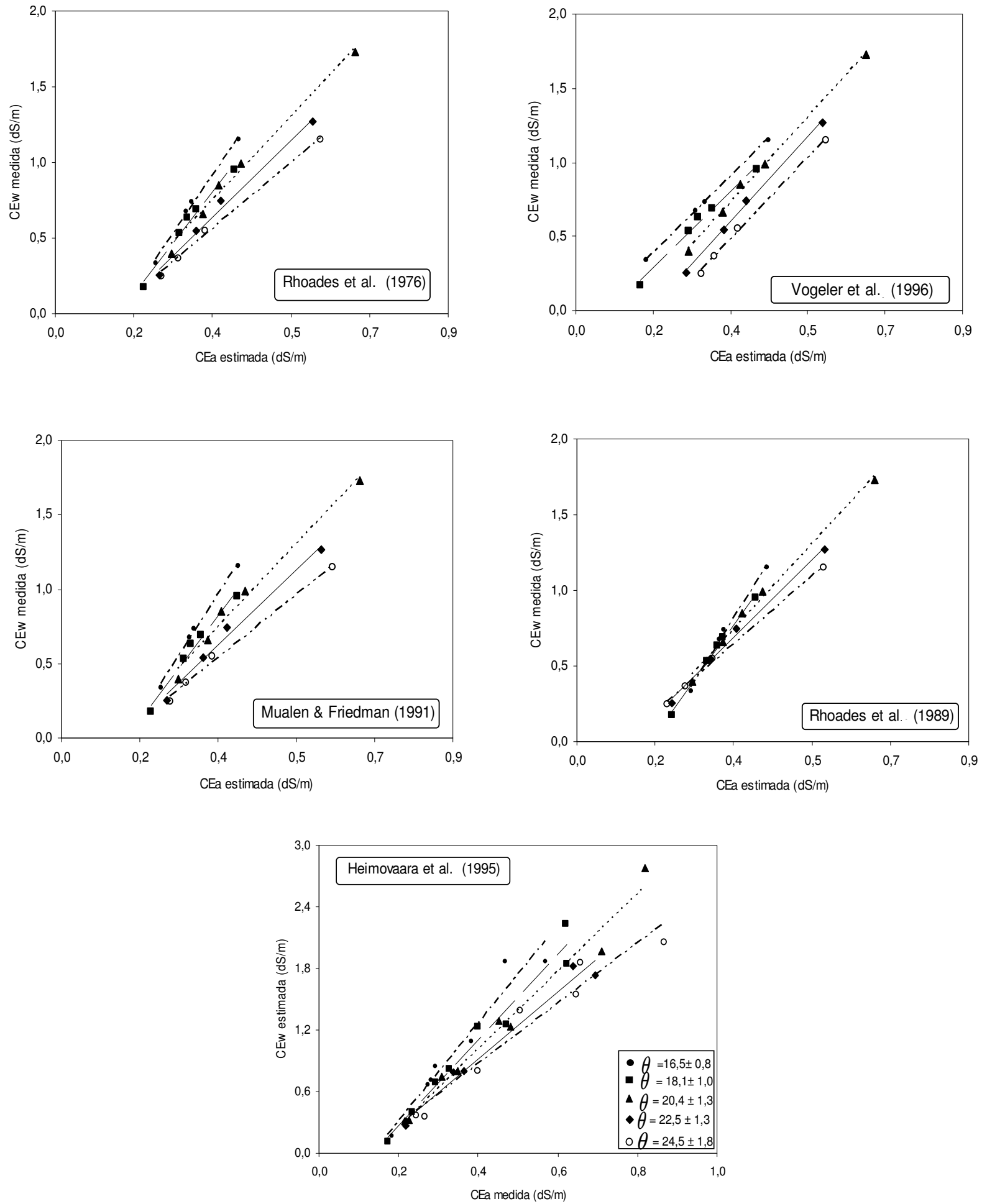

FIGURA 2. Relação entre CEa e CEw obtida a partir dos ajustes dos modelos que relacionam CEa, CEw e $\theta$, para a $\mathrm{CT}_{\text {fa }}$. 
Nas Figuras 3 e 4, referentes às $\mathrm{CT}_{\mathrm{f}}$ e $\mathrm{CT}_{\text {fa }}$, respectivamente, os dados de $\mathrm{CEa}$ estimados pelos modelos foram plotados em função dos valores medidos, estabelecendo-se uma função do tipo $\mathrm{Y}=\mathrm{aX}$. Analisando-se os coeficientes de determinação $\left(\mathrm{R}^{2}\right)$ e angulares, obtidos em relação à unidade, ou seja, a discrepância entre as retas 1:1 e estimada, percebe-se que, para a $\mathrm{CT}_{\mathrm{f}}$ (Figura 3), os modelos que proporcionaram os melhores ajustes entre dados estimados e medidos foram os de RHOADES et al. (1976) e de VOGELER et al. (1996), com $\mathrm{R}^{2}$ iguais a 0,7957 e 0,7908, e coeficientes angulares iguais a 0,9725 e 0,9720, respectivamente. O modelo de RHOADES et al. (1989) foi o que apresentou a menor relação entre os valores estimados e medidos, com $\mathrm{R}^{2}$ igual a 0,6562 e coeficiente angular igual a 0,9464 , o que implica subestimação de $5,36 \%$, em relação à reta 1:1. O modelo de NADLER et al. (1984), modificado por RHOADES et al. (1989), proporcionou $\mathrm{R}^{2}$ igual a 0,7068 e coeficiente angular igual a 1,0267 , ou seja, superestima a CEw em 2,67\% em relação à reta 1:1 (Figura 3).

Para a $\mathrm{CT}_{\mathrm{fa}}$ (Figura 4), os melhores ajustes entre dados de CEa estimados e medidos foram obtidos com os modelos de VOGELER et al. (1996), MUALEN \& FRIEDMAN (1991) e de RHOADES et al. (1976), com $\mathrm{R}^{2}$ iguais a 0,8179; 0,7623 e 0,7614 e coeficientes angulares iguais a 0,9798; 0,9752 e 0,9747, respectivamente. Assim como observado para a $\mathrm{CT}_{\mathrm{f}}$, para a $\mathrm{CT}_{\mathrm{fa}}, \mathrm{o}$ modelo de RHOADES et al. (1989) foi o que proporcionou a menor relação entre os valores estimados e medidos, com $\mathrm{R}^{2}$ igual a 0,6690 e coeficiente angular igual a 0,9628, o que implica subestimação de $3,72 \%$ em relação à reta $1: 1$.

Percebe-se, em ambas as classes texturais, discrepância entre os valores estimados e medidos inferior a 5,5\% (coeficientes angulares entre 0,9464 e 1,0267) em relação à reta 1:1, exceto para 0 modelo de HEIMOVAARA et al. (1995). Apesar de esse modelo ter proporcionado os piores coeficientes angulares $\left(0,9388\right.$ e 0,9324 para as $\mathrm{CT}_{\mathrm{f}}$ e $\mathrm{CT}_{\mathrm{fa}}$, respectivamente), o modelo de RHOADES et al. (1989) foi o que proporcionou os menores valores de $R^{2}(0,6562$ e 0,6690 para as $\mathrm{CT}_{\mathrm{f}}$ e $\mathrm{CT}_{\mathrm{fa}}$, respectivamente).

Analisando-se os coeficientes angulares e o posicionamento da reta estimada, em relação à reta 1:1, das duas classes texturais, verifica-se que tais coeficientes são inferiores à unidade e que a reta estimada posiciona-se abaixo da reta 1:1, o que sugere ligeira tendência de subestimativa da variável CEa (nos modelos que explicitam a CEa - Tabela 3). Já quanto ao modelo de NADLER et al. (1984), modificado por RHOADES et al. (1989), ajustado aos dados da $\mathrm{CT}_{\mathrm{f}}$ e que explicita a CEw, verifica-se comportamento oposto, ou seja, ligeira tendência de superestimativa da variável CEw. 

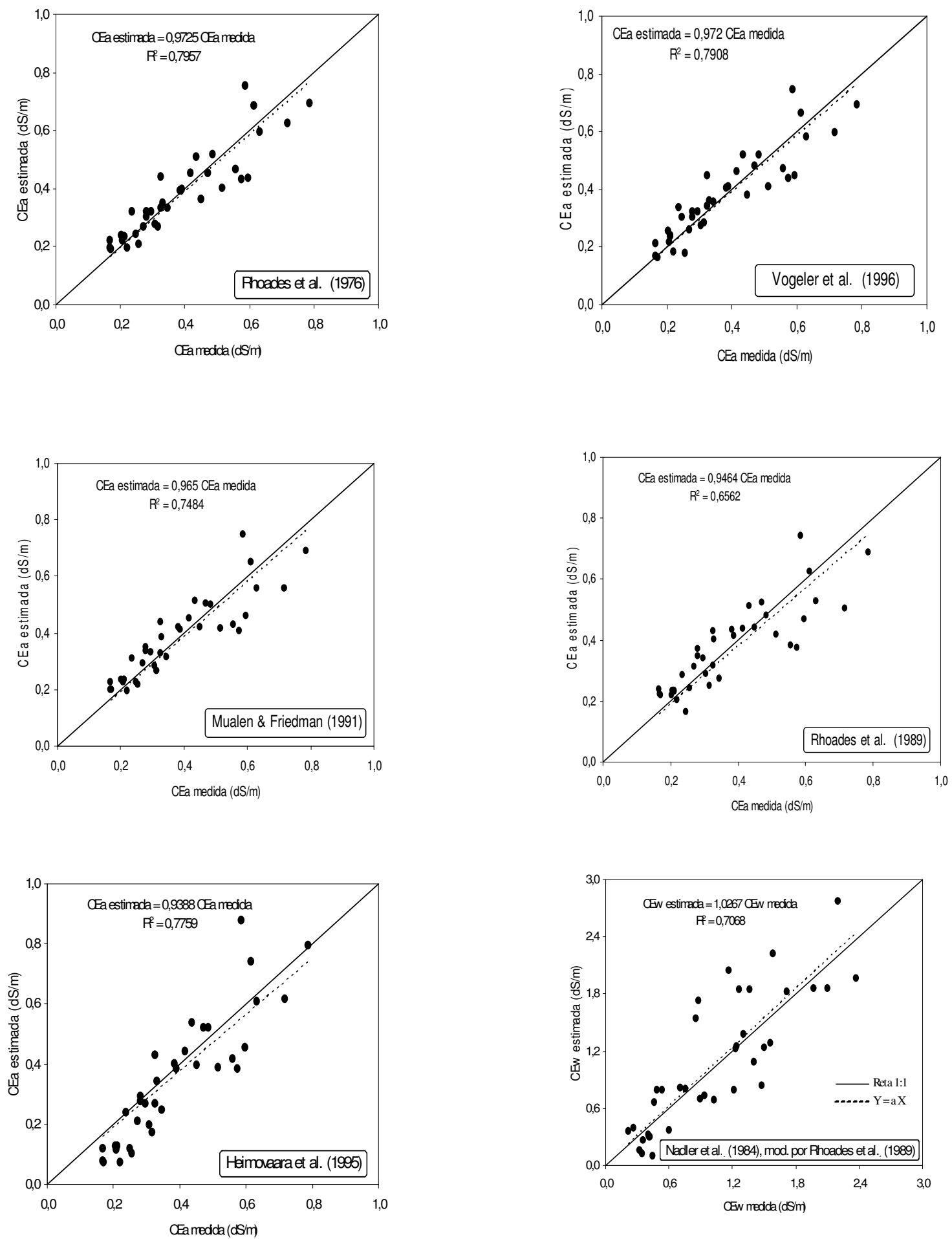

FIGURA 3. Relação entre variáveis observada e estimada pelos modelos que relacionam CEa, CEw e $\theta$, para a $\mathrm{CT}_{\mathrm{f}}$. 

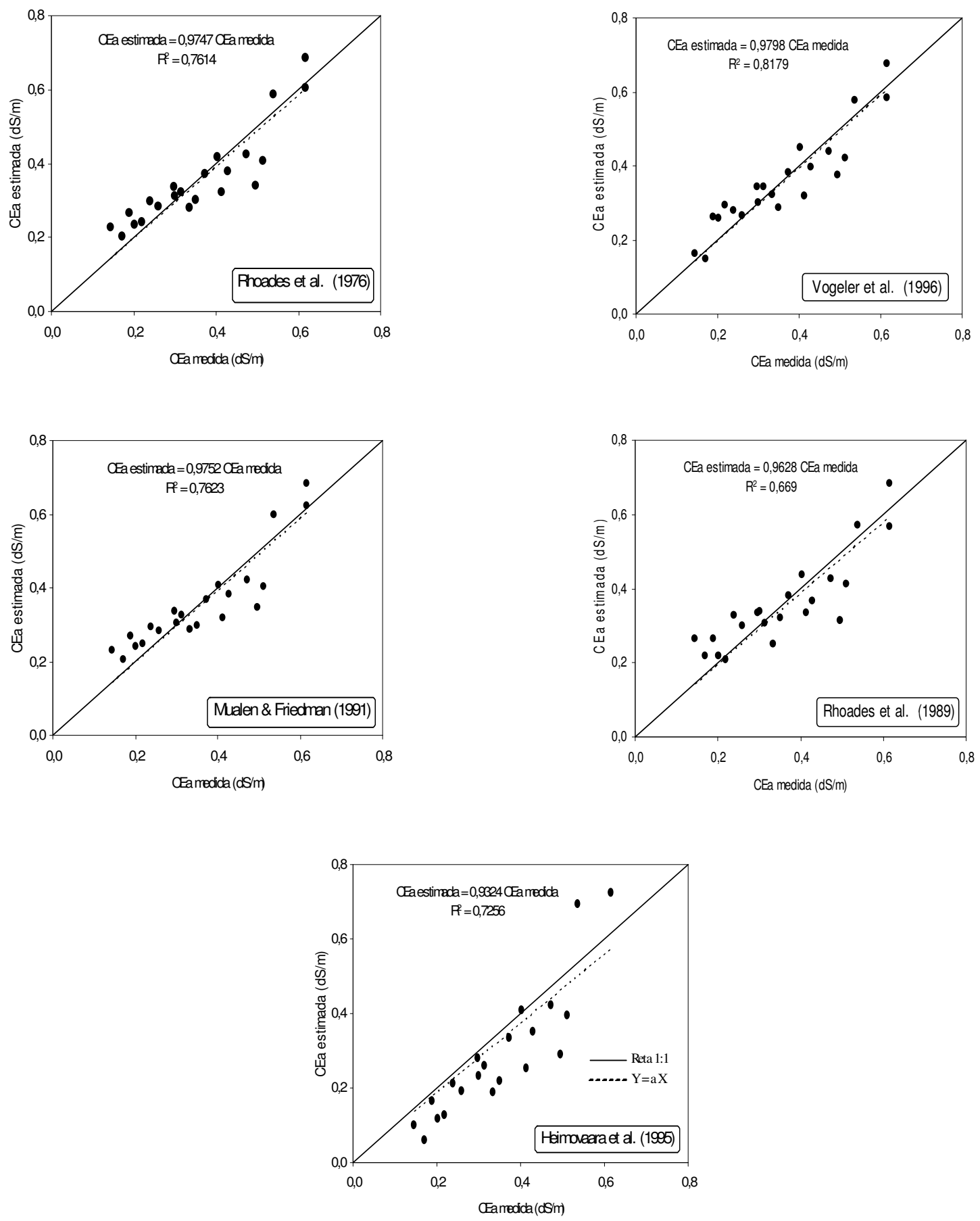

FIGURA 4. Relação entre variáveis observada e estimada pelos modelos que relacionam CEa, $\mathrm{CEw}$ e $\theta$, para a $\mathrm{CT}_{\mathrm{fa}}$.

\section{CONCLUSÕES}

Os modelos avaliados mostraram-se capazes de relacionar $\theta$, CEa e $\mathrm{CEw}$, com vistas à predição da CEw, a partir de dados de $\theta$ e CEa obtidos por meio da técnica de TDR, em condições de laboratório.

O modelo de RHOADES et al. (1976) foi o que melhor relacionou $\theta$, CEa e CEw para a classe textural franca $\left(\mathrm{CT}_{\mathrm{f}}\right)$, e o de VOGELER et al. (1996), para a classe textural franco-arenosa $\left(\mathrm{CT}_{\mathrm{fa}}\right)$, em condições de laboratório. 


\section{REFERÊNCIAS}

COELHO, E.F.; OR, D. A parametric model for two-dimensional water uptake by corn roots under drip irrigation. Soil Science Society of America Journal, Madison, v.60, n.6, p.1039-49, 1996.

DALTON, F.N.; HERKELRATH, W.N.; RAWLINS, D.S.; RHOADES, J.D. Time-domain reflectometry: Simultaneous measurement of soil water content and electrical conductivity with a single probe. Science, Washington, v.224, n.4651, p.989-90, 1984.

DE NEVE, S.; VAN DE STEENE, J.; HARTMANN, R.; HOFMAN, G. Using time domain reflectometry for monitoring mineralization of nitrogen from soil organic matter. European Journal of Soil Science, Amsterdam, v.51, n.2, p.295-304, 2000.

GIESE, K.; TIEMANN, R. Determination of the complex permitivity from the sample time domain reflectometry. Soil Science Society of America Journal, Madison, v.7, n.1, p.45-9, 1975.

HEIMOVAARA, T.J.; FOCKE, A.G.; BOUTEN, W.; VERSTRANTEN, J.M. Assessing temporal variations in soil water composition with time domain reflectometry. Soil Science Society of America Journal, Madison, v.59, n.3, p.689-98, 1995.

JACOBSEN, O.H.; SCHJONNING, P. A laboratory calibration of time domain reflectometry for soil water measurement including effects of bulk density and texture. Journal of Hydrology, Amsterdam, v.151, n.2, p.147-57, 1993.

LEDIEU, J.; De RIDDER, P.; De CLERCK, P; DAUTREBANDE, S. A method measuring soil water moisture by time-domain reflectometry. Journal of Hydrology, Amsterdam, v.88, n.1, p.31928, 1986.

MMOLAWA, K.; OR, D. Root zone solute dynamics under drip irrigation: a review. Plant and Soil, Dordrecht, v.222, n.1-2, p.163-90, 2000.

MUALEN, Y.; FRIEDMAN, S.P. Theoretical prediction of electrical conductivity in saturated and unsaturated soil. Water Resources Researches, Washington, v.27, n.10, p.2771-7, 1991.

MUÑOZ-CARPENA, R.; REGALADO, C.M.; ALVAREZ-BENEDÍ, J.; SOCORRO, A.R.; PÉREZ, N. Determinación simultánea mediante TDR del transporte de agua y un soluto salino en el suelo. In: LÓPEZ, J.J.; QUEMADA, M. (Ed.). Temas de Investigación en Zona no Saturada. Pamplona: Universidade Pública de Navarra, 2001. p.1-7.

NADLER, A. Discrepancies between soil solute concentration estimates obtained by TDR and aqueous extracts. Australian Journal Soil Researches, Canberra, v.35, n.3, p 527-37, 1997.

NADLER, A.; FRENKEL, H.; MANTELL, A. Applicability of the four-probe technique under extremely variable water contents and salinity distribution. Soil Science Society of America Journal, Madison, v.48, n.6, p.1258-61, 1984.

NOBORIO, K. Measurement of soil water content and electrical conductivity by time domain reflectometry: a review. Computers and Electronics in Agriculture, Madison, v.31, n.3, p.213-37, 2001.

PERSSON, M.; UVO, C.B. Estimating soil solution electrical conductivity from time domain reflectometry measurements using neural networks. Journal of Hydrology, Amsterdam, v.273, n.14, p.249-56, 2003.

PINTO, J.M. Fertirrigação em fruticultura irrigada. Revista ITEM, Viçosa, v.49, n.1, p.14-23. 2001. PONIZOVSKY, A.A.; CHUDINOVA, S.M.; PACHEPSKY, Y.A. Performance of TDR calibration models as affected by soil texture. Journal of Hydrology, Amsterdam, v.218, n.1, p.35-43, 1999.

RHOADES, J.D.; MANTEGHI, N.A.; SHOUSE, P.J.; ALVES, W.J. Soil electrical conductivity and salinity: new formulations and calibrations. Soil Science Society of America Journal, Madison, v.53, n.2, p.433-9, 1989. 
RHOADES, J.D.; OSTER, J.D. Solute content. In: KLUTE, A. (Ed.) Methods of soil analysis. 2.ed. Part 1. Agronomy. Madison: ASA/SSSA, 1986. p.995-1006. (Monograph 9)

RHOADES, J.D.; RAATS, P.A.; PRATHER, R.J. Effects of liquid phase electrical conductivity, water content and surface conductivity on bulk soil electrical conductivity. Soil Science Society of America Journal, Madison, v.40, n.5, p.651-5, 1976.

RICHARDS, L.A. Diagnosis and improvement of saline and alkali soils. Washington: United States Salinity Laboratory, 1954. 160 p. (Agriculture Handbook, 60)

SOUZA, C.F. A utilização da reflectometria no domínio do tempo (TDR) na modelagem do bulbo molhado do solo irrigado por gotejamento. 2002. 115 f. Tese (Doutorado em Engenharia Agrícola) - Universidade Estadual de Campinas, Campinas, 2002.

TOMMASELLI, J.T.G.; BACCHI, O.O.S. Calibração de um equipamento de TDR para medida de umidade de solos. Pesquisa Agropecuária Brasileira, Brasília, v.36, n.9, p.1145-54, 2001.

TOPP, G.C.; DAVIS, J.L. Electromagnetic determination of soil water content: measurements in coaxial transmission lines. Water Resources Researches, Washington, v.16, n.2, p.574-82, 1980.

VOGELER, I.; CLOTHIER, B.E.; GREEN, S.R.; SCOTTER, D.R.; TILLMAN, R.W.

Characterizing water and solute movement by TDR and disk permeametry. Soil Science Society of America Journal, Madison, v.60, n.1, p.5-12, 1996.

VOGELER, I.; CLOTHIER, B.E.; GREEN, S.R. TDR estimation of the resident concentration of electrolyte in the soil solution. Australian Journal Soil Researches, Canberra, v.35, n.3, p.515-26, 1997.

WARD, P.R.; DUNIN, F.X. Growing season evapotranspiration from duplex soils in south-western Australia. Agricultural Water Management, Amsterdam, v.50, n.2, p.141-59, 2001.

WRAITH, J.M.; DAS, B.S. Monitoring soil water and ionic solute distributions using time domain reflectometry. Soil and Tillage Research, Amsterdam, v.47, n.1-2, p.145-50, 1998. 